\title{
SELECTIVE RESONANCE ENHANCED MULTIPHOTON IONIZATION OF AROMATIC POLYMERS IN SUPERSONIC BEAM MASS SPECTROMETRY
}

\author{
DAVID A. LUSTIG and DAVID M. LUBMAN*
}

Department of Chemistry, The University of Michigan, 930 N. University Ave., Ann Arbor, MI 48109 (USA)

(First received 14 September 1990; in final form 9 November 1990)

\begin{abstract}
Various polymers have been studied using a pulsed laser evaporation method for volatilizing these species and entraining them into a supersonic jet expansion followed by resonance enhanced multiphoton ionization (REMPI) detection in a reflectron time-of-flight mass spectrometer. In most cases the monomer is detected as the dominant ion, although sometimes smaller characteristic fragments are observed instead. Fragmentation of the monomer can also be produced for structural analysis. However, larger oligomers are rarely observed with any intensity unless the average molecular weight distribution is less than 1000. In addition, REMPI at $266 \mathrm{~nm}$ is shown to be a means of selectively detecting absorbing aromatic polymers in polymer blends without interference from aliphatic or non-aromatic polymers. Among the polymers studied are polystyrene, EPON epoxy resins and various polyamides.
\end{abstract}

\section{INTRODUCTION}

Resonance enhanced multiphoton ionization (REMPI) has been shown to be an ionization method with unique properties for mass spectrometry [1-4]. In particular, a two-photon process known as resonant two-photon ionization (R2PI) has found wide application to problems in chemical analysis. The R2PI process involves the absorption of two photons by a molecule where the first photon excites the species to an intermediate electronic state and absorption of a second photon results in ionization. The sum of the two photons must therefore exceed the ionization potential of the molecule. However, for most aromatics studied to date the excess energy deposited in the molecule in the ionization process remains low when near-ultraviolet photons are used. The result is that R2PI can produce relatively soft ionization where either only the molecular ion or minimal fragmentation is observed for identification. In addition, with an increase of the laser beam power density, extensive

* Author to whom correspondence should be addressed. 
fragmentation may be induced for structural analysis. The fragmentation can be varied simply by changing the laser intensity, and, at high input energy, fragments as small as $\mathrm{C}^{+}$have been observed [5-7]. Thus relatively versatile fragmentation can be induced for structural analysis and identification.

The feature that makes R2PI a unique ionization source for mass spectrometry is the potential for wavelength selectivity. Although ions are detected as the final product, the ionization signal reflects the absorption cross-section of the resonant intermediate state. Thus, selectivity can be based on either the relative absorptions or ionization potentials of molecules using a single wavelength. In previous experiments, increased selectivity has been achieved by tuning the wavelength to produce an optical-ionization spectrum characteristic of a species [8-11]. The key point here is that R2PI can provide selective detection of target molecules in the presence of a background matrix.

The use of R2PI has generally been limited to reasonably volatile species. However, in recent work [1-4,8-10] R2PI has been used for detection of non-volatile molecules using a pulsed laser desorption technique. The laser desorption technique involves using a pulsed laser to induce a rapid heating effect in which the molecules desorb from a surface before they have time to decompose. In the desorption process, both ions and neutrals are formed in a ratio that depends to a first approximation on the surface temperature induced by the laser. However, since the bulk of the species desorbed are neutral, a two-step process involving desorption of neutral molecules with subsequent ionization by R2PI will prove to be a much more efficient process for most applications. In addition, the separation of the desorption and ionization steps allows for the possibility of selective detection in the ionization process based upon R2PI.

In this work, we investigate the R2PI of several aromatic polymers desorbed into the gas phase using a pulsed $\mathrm{CO}_{2}$ laser. The neutral species are entrained into a supersonic jet expansion and then swept into the acceleration region of a reflectron time-of-flight mass spectrometer (TOF-MS) where R2PI is produced. The capability of R2PI for detection of monomer units from the polymer is studied. We demonstrate that one can identify polymers based upon the molecular ion of the monomer unit produced by R2PI and/or the fragmentation that results upon absorption of subsequent photons. In addition, the selective detection of aromatic polymers in polymer blends is discussed.

\section{EXPERIMENTAL}

The experimental set-up is shown in Fig. 1. It consists of a reflectron time-of-flight mass spectrometer (RETOF-MS) equipped with a pulsed valve molecular beam source (R.M. Jordan Company, Mountain View, CA), a $\mathrm{CO}_{2}$ 


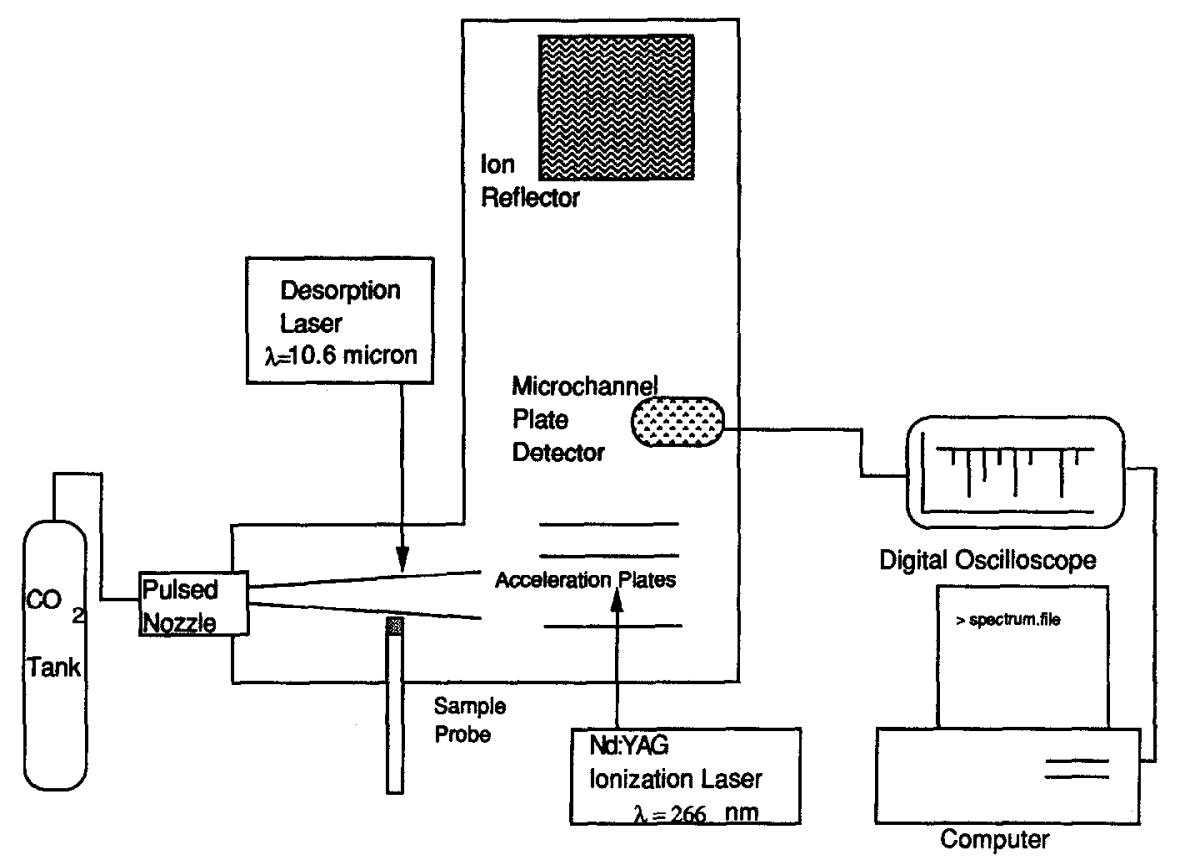

Fig. 1. Schematic of the experimental apparatus.

desorption laser (modified Quanta Ray EXC-1 excimer laser) and a Nd : YAG laser (Spectra Physics DCR-3) for ionization. The reflectron mass spectrometer is constructed of a 6 in diameter stainless steel cross which serves as the ionization chamber and an 8 in diameter stainless steel flight tube. Ions created in the chamber are accelerated into the flight tube at $2-3 \mathrm{kV}$. At the reflector the ions are turned back toward the source with an angular displacement from the initial flight axis. The ions are then detected by a two-stage microchannel plate detector. The RETOF-MS is pumped by two diffusion pump stacks. Each stack consists of a gate valve, a liquid nitrogen cold trap and a diffusion pump. A 6 in pump is used on the ionization chamber and a 4 in pump is used on the flight tube. Additional pumping is provided to the chamber via a liquid nitrogen cold baffle mounted on the cross. This baffle pumps away the $\mathrm{CO}_{2}$ carrier gas from the jet. Typically the pressure in the flight tube is $2 \times 10^{-6}$ Torr and the pressure in the ionization region is slightly higher.

Laser desorption (LD) of the sample is accomplished in the following manner. The LD probe, with a Macor ceramic tip for the desorption surface, is introduced through a vacuum lock into the ionization chamber on axis with the pulsed valve and directly above the ionization region. The $10.6 \mu \mathrm{m}$ desorption beam produced by the $\mathrm{CO}_{2}$ laser enters the mass spectrometer through an $\mathrm{NaCl}$ window that is located opposite the LD probe. The desorption beam 
is externally focused to a small spot on the LD probe tip. The power density of the desorption beam is approximately $5 \times 10^{6} \mathrm{~W} \mathrm{~cm}^{-2}$. The desorbed neutrals are swept into the ionization region by the molecular beam emitted by the pulsed valve. The neutrals are ionized by a $266 \mathrm{~nm}$ laser beam produced as the fourth harmonic of the Nd:YAG laser. This output is reduced to a $2 \mathrm{~mm}$ collimated beam through the combination of a concave lens and a convex lens. Typical operation of the Nd: YAG laser is at $10 \mathrm{~Hz}$ at a power density of $5 \times 10^{5}-1 \times 10^{7} \mathrm{~W} \mathrm{~cm}^{-2}$.

It should be noted that this experiment could also be performed without the use of the supersonic jet. However, the jet enhances the performance of the experiment in several ways. The use of the jet allows the physical separation of the desorption from the ionization process. If ions are produced in the desorption process, then these ions will be deflected by the electric fields before they reach the acceleration region of the RETOF-MS and are not detected. Only neutral species are transported by the jet into the RETOF-MS for detection. In addition, the jet expansion is used to enhance the resolution of the RETOF device owing to the translational cooling and the directed motion of the molecules in the jet as compared with a thermal expansion. The resolution of the RETOF-MS is 2300 at $\mathrm{m} / \mathrm{z} 93$ using the jet expansion.

The desorption event and the valve pulsing are triggered by the Nd:YAG laser flashlamp oscillator trigger monitor with appropriate delays to coincide with the $\mathrm{Nd}$ : YAG light pulse. When timed properly, the $\mathrm{CO}_{2}$ laser fires such that the maximum number of resulting neutrals are entrained into the early portion of the jet pulse profile. This seeded portion of the molecular beam is synchronized with the $266 \mathrm{~nm}$ light pulse in the acceleration region of the RETOF-MS. The mass spectra are recorded with a LcCroy 9400 digital oscilloscope which is interfaced to an IBM compatible computer for data storage. The digital oscilloscope is equipped with a signal averaging function. All spectra presented result from a single laser shot or the average of 50 laser shots.

Several methods of sample preparation were used depending on the nature of the analyte. The polystyrene GPC standard $(\mathrm{MW} \approx 600)$ was a tacky solid-stiff liquid which was applied directly to the probe tip as a thick film. This method provided excellent shot-to-shot stability, allowing for analysis of the same sample for several hours. The next best method involved melting the polymer onto the Macor tip. This was accomplished by heating the Macor tip with a heat gun and pressing it down into the solid sample. The sample melted onto the tip which was then cooled resulting in a film of sample that is visible to the naked eye. This method worked well for polystyrene and the polystyrene-containing blend and for the EPON resins. When the melting point of a sample was too high, other methods were used. For poly(9-vinyl carbazole) and the polyamic acid samples, the crystalline sample was made into a 
paste-like mixture with methanol. This paste was smeared onto the LD tip and the methanol was allowed to evaporate, leaving the sample clinging to the Macor. Another method was used in the case of the polymer KJB-175A which had a very high melting point and was not soluble in common solvents. In this case, a small amount of the crystalline solid was placed onto the LD probe tip. The crystalline material was pressed into a powder with a metal spatula. A small drop of glycerol was added to the powder and the sample was analyzed as such.

Four separate samples of polystyrene were analyzed. One was a polystyrene standard with an average molecular weight of 3100 (Polymer Laboratories, Stretton, UK). Two others were identical blends which were made up of three separate polymers provided by Shell Oil Company. Two of the polymers in the blend were completely aliphatic. The third was a copolymer containing styrene and an aliphatic residue. Both blend samples contain compounds added in small amounts to give the blend stability. Each blend contains the same two additives, but in different amounts. These additives are aromatic in nature. In addition, a standard polystyrene sample with an average molecular weight of 600 was provided by Polysciences Inc. (Warrington, PA). This sample is a GPC standard which is commercially available. Poly(9-vinyl carbazole) was obtained commercially from Aldrich. Several amide samples were synthesized by the Paul Rasmussen group at the University of Michigan and other polyamide samples were provided courtesy of Dr. W. Weber of Ciba-Geigy Corp. (Ardsley, NY 10502).

\section{RESULTS AND DISCUSSION}

Figures 2 and 3 show REMPI induced mass spectra of a polystyrene standard ( $M W=3100$ ) obtained from Polymer Laboratories and a polymer blend provided by Shell Development Co. In these experiments the polystyrene samples were vaporized by pulsed $\mathrm{CO}_{2}$ laser vaporization into a supersonic expansion of $\mathrm{CO}_{2}$ carrier gas and ionization was performed at $\lambda=266 \mathrm{~nm}$. The REMPI mass spectra obtained from both samples are essentially identical. The key feature is that the dominant ion peak at $\mathrm{m} / \mathrm{z} 104$ corresponds to that of the monomer unit, styrene, obtaincd at a power density of $2 \times 10^{6} \mathrm{~W} \mathrm{~cm}^{-2}$ (Fig. 3). As the laser power density is increased to $1 \times 10^{7} \mathrm{~W} \mathrm{~cm}^{-2}$ the various $\mathrm{C}_{n} \mathrm{H}_{n}^{+}$fragments characteristic of the structure of styrene are observed (as shown in Fig. 2). In Fig. 3 the laser induced REMPI mass spectra of polystyrene at lower laser power where mainly the molecular ion of the monomer is observed are shown over an extended mass range. At times small peaks possibly corresponding to higher repeat units are observed, but never those typical of the molecular weight distribution. However, a significant point is that in the Shell polymer sample only the aromatic styrenc 
SIGNAL

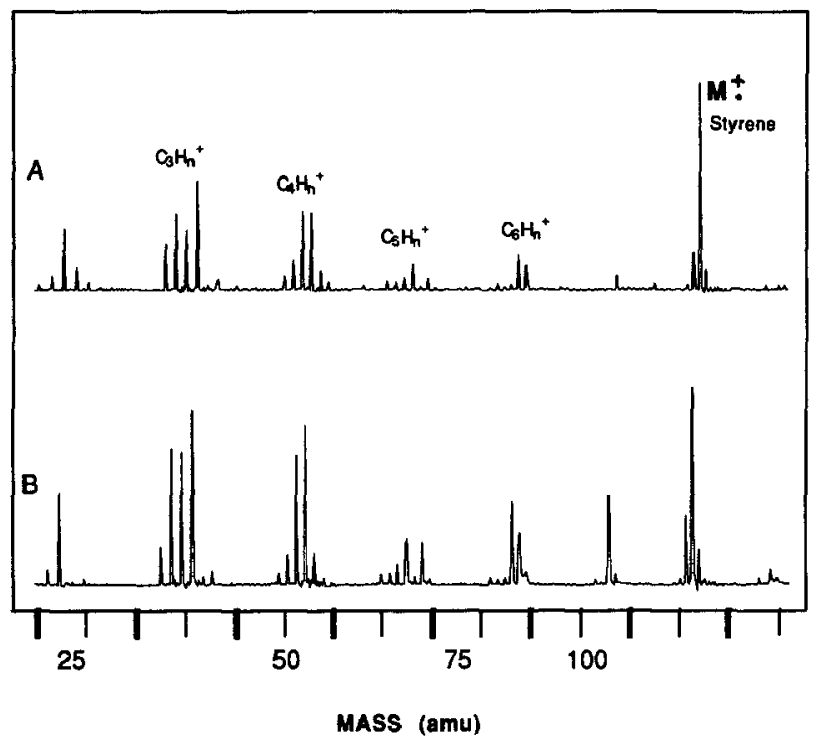

Fig. 2. Laser desorption-laser REMPI (266 nm) mass spectra of polystyrene: (A) Shell blend; (B) standard $(\mathrm{MW}=3100)$.

\section{POLYSTYRENE CONTAINING BLEND AND POLYSTYRENE}

SIGNAL

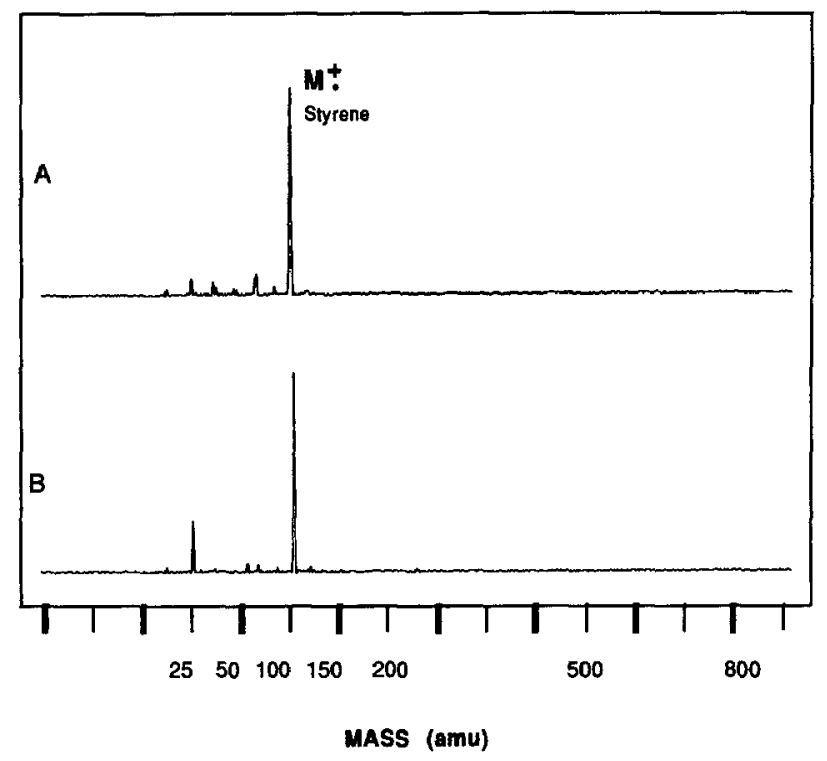

Fig. 3. Laser desorption-laser REMPI ( $266 \mathrm{~nm}$ ) mass spectra of polystyrene up to $800 \mathrm{u}$ of: (A) Shell blend; (B) standard. 
is observed, although this sample is a polymer blend mixture including at least three other aliphatic polymers. Thus, selective detection of target polymers can be achieved in polymer mixtures via laser REMPI based upon the absorption of the target polymer, while the aliphatic polymers remain transparent to the laser radiation. Several polymer blends provided by Shell were studied by REMPI at $266 \mathrm{~nm}$ and each laser induced ionization mass spectrum resulted in detection of the monomer unit molecular ion at $\mathrm{m} / z 104$ without interference from the aliphatic polymers.

In contrast, Fig. 4 shows the LD-REMPI mass spectrum of the polystyrene GPC standard obtained at $266 \mathrm{~nm}$ at a power density $P=3 \times 10^{6} \mathrm{~W} \mathrm{~cm}^{-2}$. In this case a distribution of oligomers is observed at $n=1$ to 4 , where, as shown in the accompanying structure, the total number of styrenes $=n+2$. In addition, an $n$-butyl styrene ion is observed at $m / z 162$ and also the styrene monomer, which is not shown in this figure. Molecular weight averages and the polydispersity were determined from this spectrum. The weight average, $M_{\mathrm{w}}=\Sigma N_{i} M_{i}^{2} / \Sigma N_{i} M_{i}$, was found to be $499 \mathrm{u}$. The number average, $M_{n}=\Sigma N_{i} M_{i} / \Sigma N_{i}$, was found to be $487 \mathrm{u}$. Because the sample analyzed was from a very old batch, Polysciences Inc. could not provide data for com-

POLYSTYRENE (Average MW 600)
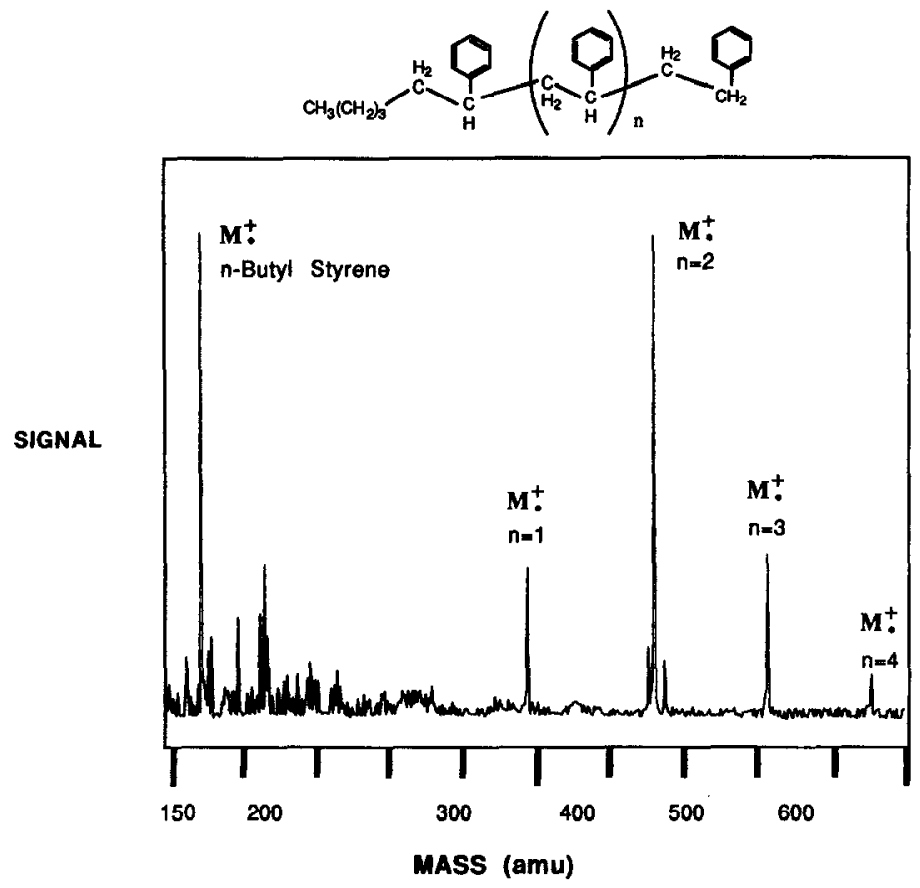

Fig. 4. Laser desorption-laser REMPI $(266 \mathrm{~nm})$ mass spectrum of polystyrene 600 $\left(3.0 \mathrm{MW} \mathrm{cm}^{-2}\right.$ ). 
parison with these results. However, data were available for their most recent batch where $M_{w}$ was determined by light scattering to be $517 \mathrm{u}$ and $M_{n}$ was determined by NMR and vapor pressure osmometry to be $504 \mathrm{u}$ and $503 \mathrm{u}$ respectively. Although the weight averages vary it is interesting to note that the polydispersity, $N=M_{w} / M_{n}$, was found to be 1.026 by LD-REMPI which is in excellent agreement with $N=1.027$ for the recent batch. A polydispersity near unity suggests that the polymer was prepared by an anionic initiator such as $n$-butyl lithium. This is consistent with our results in which a peak is seen for butyl styrene and all oligomers contain a butyl styrene terminating group. Similar laser induced REMPI distributions for polystyrene of $\mathrm{MW}<1000$ have also recently been observed in other work [12].

It should be noted in comparison that laser photoionization mass spectrometry of polystyrene vaporized by several different methods has been studied by a number of research groups, and generally the molecular ion of the monomer was detected with only a few ion peaks of low abundance to higher mass. Feldmann et al. [13] for example, used $193 \mathrm{~nm}$ radiation produced by an ArF excimer laser for photoablation of polystyrene with detection via VUV photoionization at $118.4 \mathrm{~nm}(10.5 \mathrm{eV})$ in a TOF-MS. The vaporization in this case is thought to occur via a photochemically induced bond breaking process in the bulk materials producing a high pressure gaseous plume and resulting in little of the incident laser energy remaining in the substrate [14]. This is compared with longer wavelength vaporization where substantial thermal heating may be present. In this work the $\mathrm{M}^{+}$. $(\mathrm{m} / \mathrm{z} 104)$ ion of polystyrene appeared as a strong ion peak, whereas only a negligibly small dimer peak was observed $(\mathrm{m} / \mathrm{z} 208)$. In related work Stuke and Larciprete [15] used a $248 \mathrm{~nm} \mathrm{KrF}$ laser for photoablation from polystyrene with detection via a picosecond laser $(290 \mathrm{~nm}, 1 \mathrm{ps})$ in a TOF-MS. The use of picosecond laser ionization is significant in that it may prevent fragmentation induced by the ladder-switching process using nanosecond laser radiation [16]. In the present work the monomer species $(m / z 104)$ was observed as the dominant ion peak, although species heavier than the monomer but of lower molecular weight than the dimer were detected. These species could not be attributed to simple direct fragments of the polymer chains, but rather were due to fast chemistry and/or photochemistry during the ablation process. In other work, ion beam sputtering or electron stimulated desorption has been used to study bulk polystyrene. In the work of Pallix et al. [17] single photon ionization using pulsed coherent VUV radiation at $118 \mathrm{~nm}$ was used to detect polystyrene desorbed via these methods in a reflectron TOF-MS. Once again the monomer was detected with considerable intensity accompanied by other fragments at lower mass due to fragmentation of the monomer. Small ion peaks up to $\mathrm{m} / \mathrm{z} 265$ were also observed but no distribution of larger oligomers was observed. In these experiments the $\mathrm{CO}_{2}$ laser desorption power 


\section{EPON EPOXY RESINS}

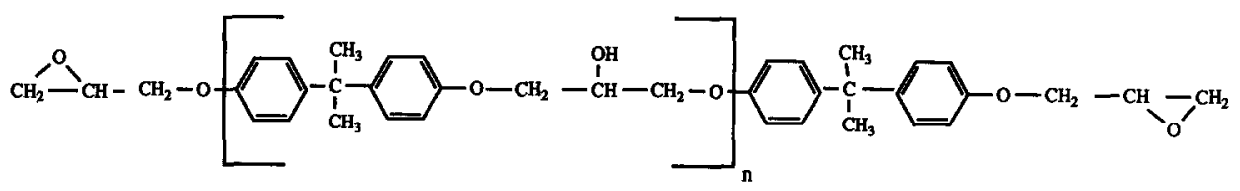

Fig. 5. Structure of EPON epoxy resins.

was varied over the range $5 \times 10^{5} \mathrm{~W} \mathrm{~cm}^{-2}-5 \times 10^{7} \mathrm{~W} \mathrm{~cm}^{-2}$ with no significant effect upon the mass spectrum. Even at the lowest desorption power used, no enhancement in the detection of larger oligomer units was observed. Similar results were also observed by Hrubowchak and Winograd [18] where ion sputtering followed by REMPI at $266 \mathrm{~nm}$ detected essentially monomer species in a TOF device. Thus, it appears that large oligomers desorbed from the bulk either photochemically or thermally decompose upon desorption whether laser vaporization or particle sputtering is utilized. Polymer distributions have been observed in thin films using various methods [19]. We attempted to desorb polystyrene from thin films prepared on the heated probe tip by merely passing the tip across the surface of the sample rather than immersing it into the sample. These films are estimated to be $\simeq 1 \mu \mathrm{m}$ thick. However, only small signals were observed and insufficient intensity was available to observe any oligomers at higher mass.

Several other polymer samples were studied including EPON resins provided by Shell Development (Westhollow, TX). Two different EPON resins with various additives were studied. In general, EPON resin structures of these samples are shown in Fig. 5. The samples were desorbed by pulsed $\mathrm{CO}_{2}$ laser radiation over a range $P=5 \times 10^{5}-5 \times 10^{7} \mathrm{~W} \mathrm{~cm}^{-2}$ into the jet expansion of $\mathrm{CO}_{2}$ carrier gas and ionized by $266 \mathrm{~nm}$ radiation in the TOF-MS. The REMPI mass spectrum of EPON resin A is shown in Fig. 6 obtained at a laser power of $P \approx 2 \times 10^{6} \mathrm{~W} \mathrm{~cm}^{-2}$. The dominant ion peak is the $n=0$ ion at $\mathrm{m} / z 325$ (see Fig. 7). In addition, several small peaks at higher signal are observed which appear to correspond to the $(M-15)^{+}$ions of $n=2,4,5$ and 8 (Table 1). It is known from the chromatographic separation of oligomers of

\section{TABLE 1}

Oligomers for EPON epoxy resins

\begin{tabular}{ll}
\hline Mass observed & Ion \\
\hline 893 & $M-15$ for $n=2$ \\
1461 & $M-15$ for $n=4$ \\
1745 & $M-15$ for $n=5$ \\
2597 & $M-15$ for $n=8$ \\
\hline
\end{tabular}




\section{EPON RESIN A}

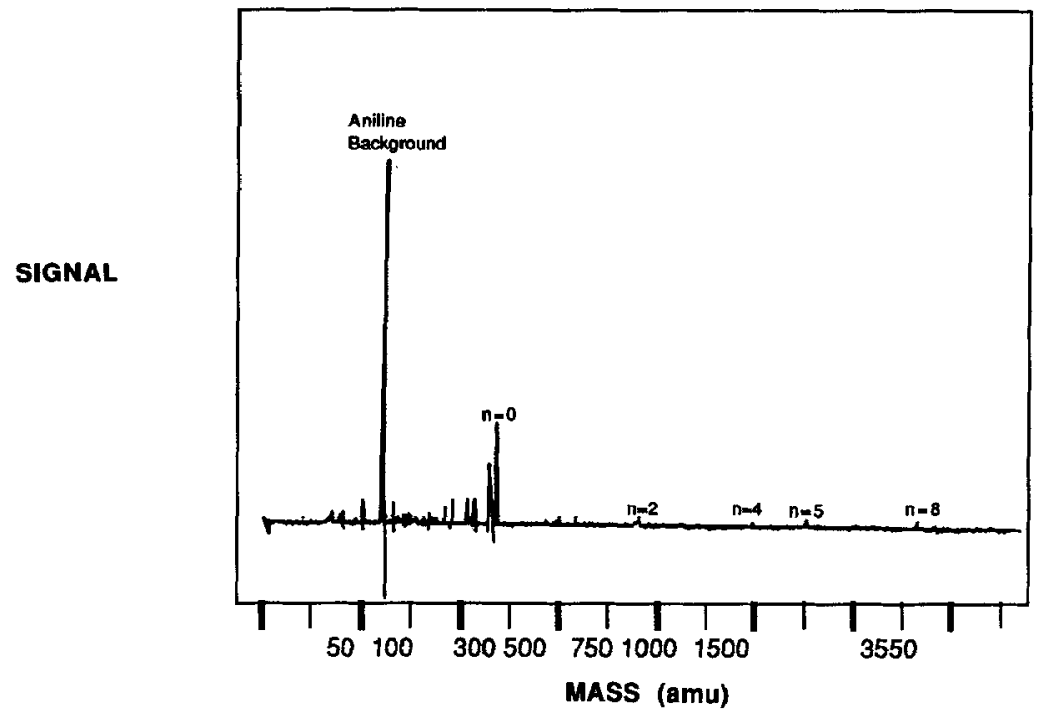

Fig. 6. Laser desorption-laser REMPI $(266 \mathrm{~nm})$ mass spectrum of EPON resin A.

ERAGMENT

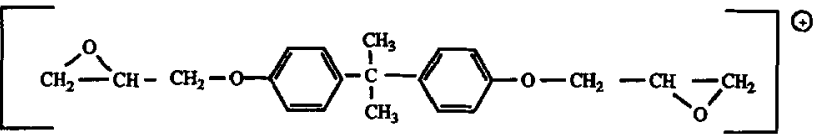

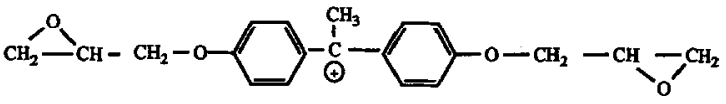<smiles>CC(C)(c1ccc(O)cc1)c1ccc(O)cc1</smiles><smiles>CC(C)(O)c1ccc(OC[C]2CO2)cc1</smiles><smiles>CC(C)(C)C1CC2CCC1CC2(O)O</smiles><smiles>[CH2-]C(=O)c1ccc(O)cc1</smiles>

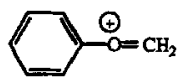

MASS

340

107

Fig. 7. Structure of $n=0$ ion of EPON resin and various fragment ions induced by laser REMPI. 
these samples [20] that these species are indeed present, although the actual size distribution of oligomers in this sample is unknown. In addition, as shown in Fig. 7, fragmentation can be induced in these polymers with fragments that are clearly assignable to structural units characteristic of the EPON resin. The fragments obtained are very similar to that obtained by fast atom bombardment mass spectrometry (FAB-MS) [21] for monofunctional epoxy which has a very similar structure to the EPON resin studied herein. In the FAB study, the usual proton addition mechanism was barely observed and the radical cation was present. The result was an electron-impact-like mass spectrum which is also observed in REMPI since the radical cation is the dominant peak. In addition, the polymer additives were not observed by REMPI in EPON resins A or B and the two samples thus provided very similar spectra. Laser induced REMPI was also studied for both the EPON resins and polystyrene samples at $213 \mathrm{~nm}$ (fifth harmonic of the Nd: YAG laser) and $193 \mathrm{~nm}$ (ArF excimer laser). At $213 \mathrm{~nm}$ the monomer units were generally observed as at $266 \mathrm{~nm}$, although higher oligomers were never observed. At $193 \mathrm{~nm}$ extensive fragmentation of the monomer was observed even at the lowest laser power used $\left(P \approx 1 \times 10^{5} \mathrm{~W} \mathrm{~cm}^{-2}\right)$. At lower energy $(280 \mathrm{~nm})$ the monomer units were

POLYAMIC ACID MODEL COMPOUND<smiles>O=C(O)c1cc(C(=O)O)c(C(=O)Nc2ccc(Oc3ccccc3)cc2)cc1C(=O)Nc1ccc(Oc2ccccc2)cc1</smiles>

$\mathrm{C}_{34} \mathrm{H}_{24} \mathrm{~N}_{2} \mathrm{O}_{3}$

588 a.m.u.

POLYAMIC ACID

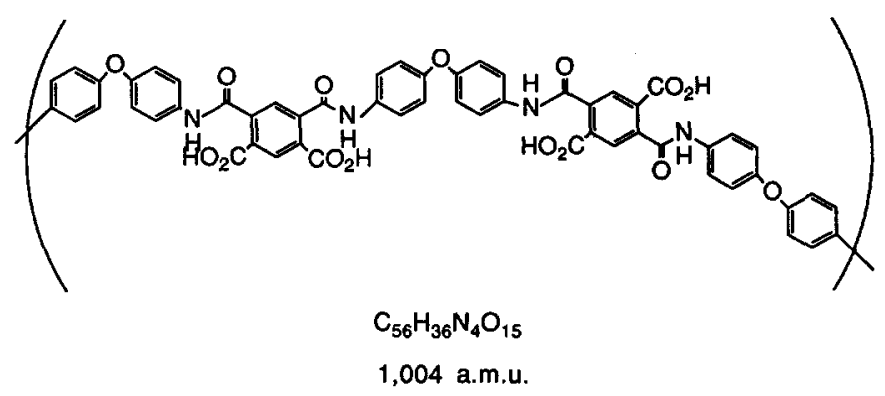

Fig. 8. Structure of polyamic acid model compound and polyamic acid. 
POLYAMIC ACID MODEL COMPOUND

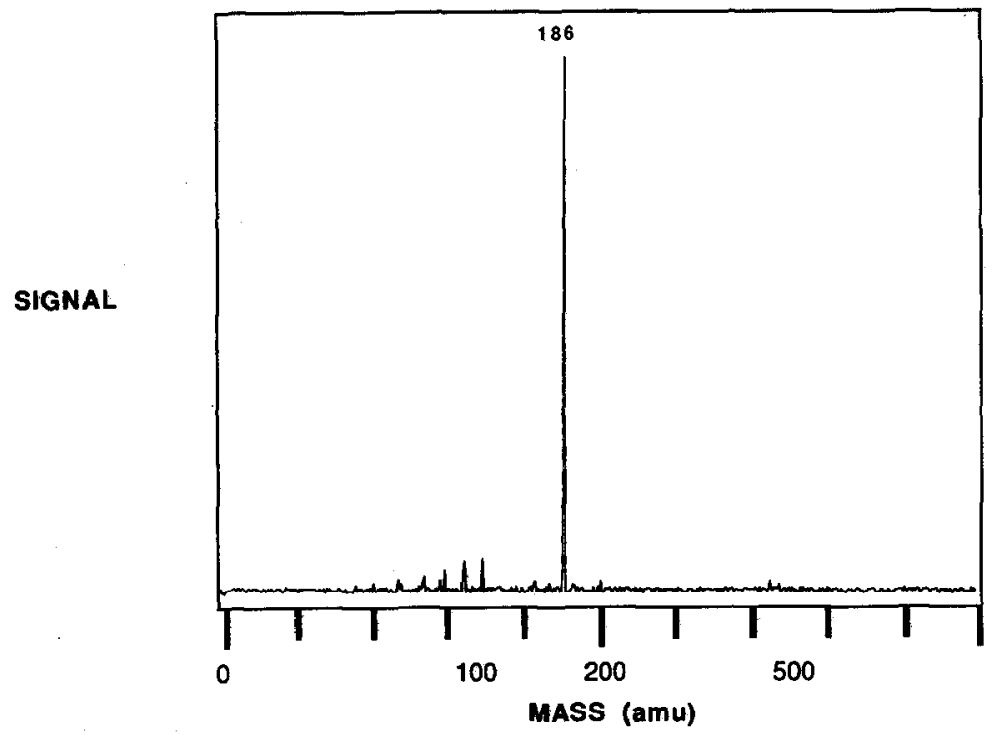

Fig. 9. Laser desorption-laser REMPI $(266 \mathrm{~nm})$ mass spectrum of polyamic acid model compound.

\section{POLYAMIC ACID}

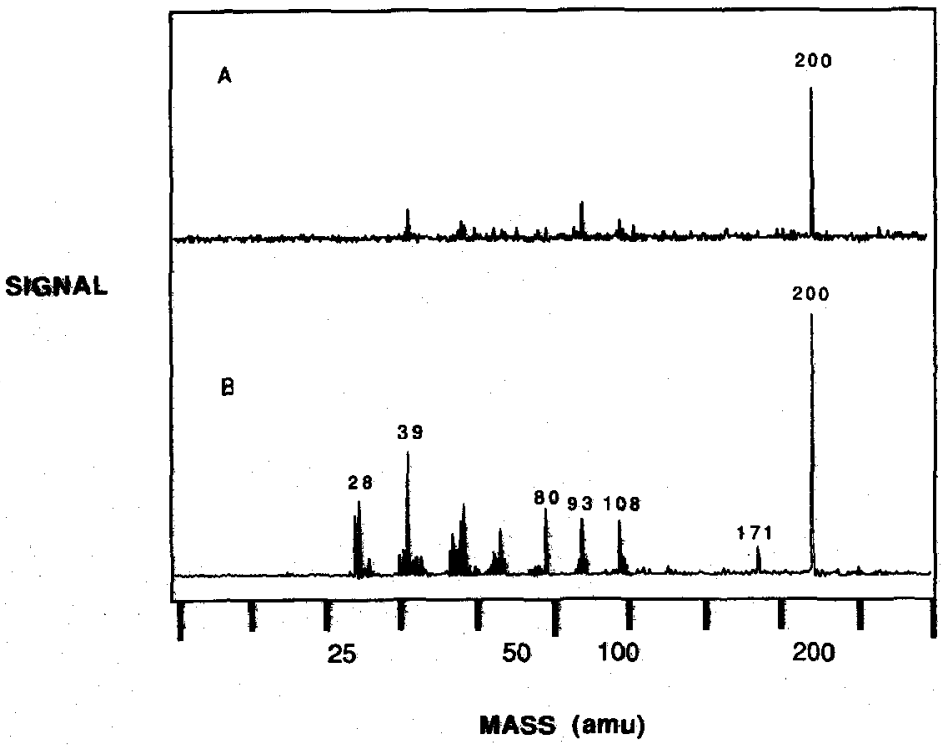

Fig. 10. Laser desorption-laser REMPI $(266 \mathrm{~nm})$ mass spectra of polyamic acid: (A) $2 \mathrm{MW} \mathrm{cm}^{-2}$; (B) $5 \mathrm{MW} \mathrm{cm}^{-2}$. 
MODEL COMPOUND FRAGMENT

186 a.m.u.<smiles>Nc1ccc(Oc2ccccc2)cc1</smiles>

\section{POLYAMIC ACID FRAGMENTS}

200 a.m.u.<smiles>CCc1ccc(Oc2ccc(N)cc2)cc1</smiles>

171 a.m.u.<smiles>c1ccc(Oc2ccccc2)cc1</smiles>

108 a.m.u.<smiles>Oc1ccncc1</smiles>

and/or<smiles>COc1ccc(N)cc1CC1CC(N)C=CC1=O</smiles>

93 a.m.u.<smiles>Nc1ccccc1I</smiles>

80 a.m.u.<smiles>c1ccncc1</smiles>

Fig. 11. Proposed fragment ions for peaks obtained in the laser REMPI induced fragmentation of polyamic acid in Fig. 10B.

distinctly observed; however, the much lower energy available from the frequency doubled dye laser, as opposed to the fourth harmonic of the Nd:YAG laser, provided much less versatility in inducing fragmentation.

Polyamic acid and a polyamic acid model compound obtained courtesy of Dr. W. Weber of Ciba-Geigy were also studied. The structures of these compounds are shown in Fig. 8. The REMPI mass spectrum $(266 \mathrm{~nm})$ of the model compound obtained by laser desorption-entrainment into a jet of $\mathrm{CO}_{2}$ carrier is shown in Fig. 9. At a laser power of $1 \times 10^{6} \mathrm{~W} \mathrm{~cm}^{-2}$, the highest mass ion observed is a fragment at $\mathrm{m} / z \mathbf{z}$ 186. No molecular ion of the monomer is observed. The REMPI mass spectrum of polyamic acid is shown in Fig. 10. The highest mass ion observed is a fragment at $\mathrm{m} / \mathrm{z} 200$ and the corresponding structure is shown in Fig. 11. As the power is increased to $5 \times 10^{6} \mathrm{~W} \mathrm{~cm}^{-2}$, 


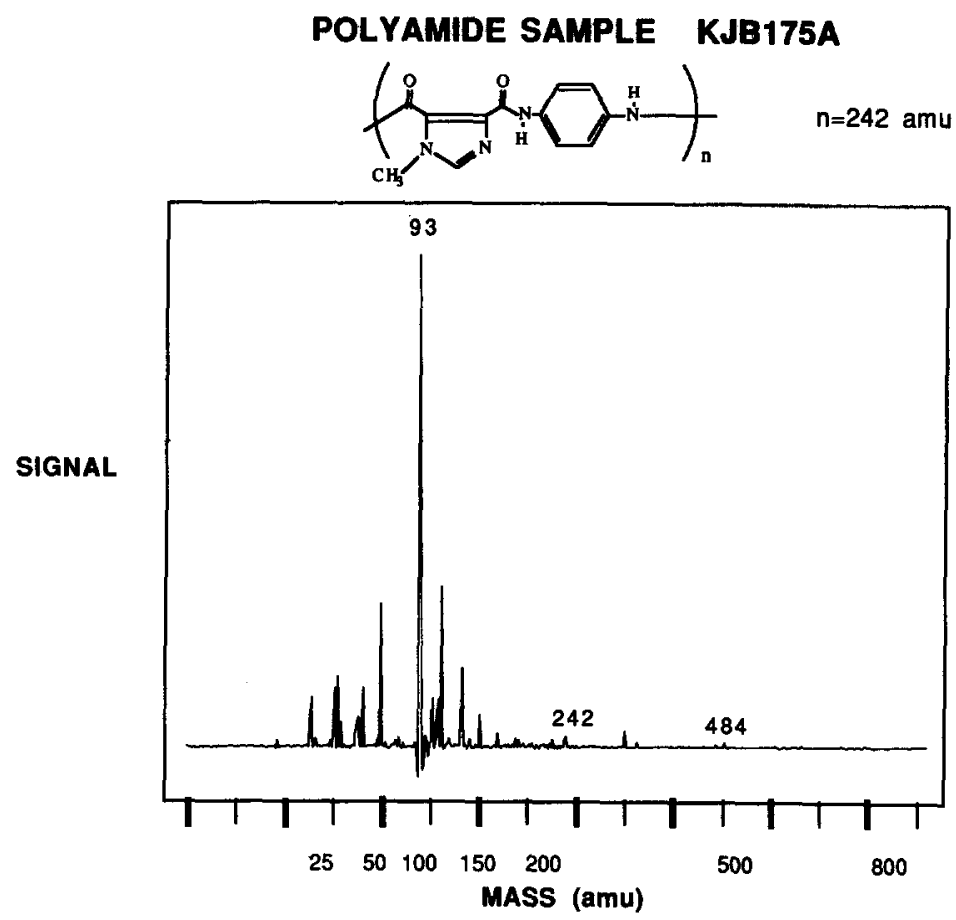

Fig. 12. Laser desorption-laser REMPI ( $266 \mathrm{~nm})$ mass spectrum of KJB 175A polyamide.

fragmentation is observed and the proposed structure of these fragments is shown in Fig. 11. In this case though, it is not clear whether fragmentation is due to the desorption or ionization process. It is significant to note that both the polyamic acid and the model compound produce fragments via cleavage of the amide bond (as expected) with retention of the amines. Thus, the model compound is useful for predicting the mass spectrum of the polymer. REMPI at $266 \mathrm{~nm}$ was also applied to KJB175A-polyamide prepared by the group of P.G. Rasmussen at the University of Michigan as shown in Fig. 12. In this case the monomer unit at $m / z=242$ is observed at a laser power $P=2 \times$ $10^{6} \mathrm{~W} \mathrm{~cm}^{-2}$. In addition, a minor peak due to $n=2$ is observed as well as various fragments of the monomer unit which are observed as the major peaks of the spectrum. Other related polyamides were studied and similar results were obtained in each case. Other related compounds studied by REMPI at $266 \mathrm{~nm}$ include poly(9-vinyl carbazole). Similar results were obtained here, but only minor peaks at best were observed for higher repeating units.

In conclusion, laser evaporation-laser REMPI at $266 \mathrm{~nm}$ serves as an excellent method for detection of aromatic polymers. This in turn can allow for identification of unknown polymers via relatively soft ionization resulting in production of the molecular ion of the monomer or some diagnostic 
fragment. In addition, further fragmentation resulting in $\mathrm{C}_{n} \mathrm{H}_{n}^{+}$cluster fragments of the monomer can be produced by increasing the laser power. However, larger oligomer units are rarely observed with any significant intensity. This appears to be due to photodecomposition or thermal decomposition of the polymer in the bulk material due to the desorption process. However, in polymers with an average molecular weight less than $1000 \mathrm{u}$, various repeat units can sometimes be observed as in the case of polystyrene. Thus, at present, this is not a successful technique for determination of molecular weight distributions. However, the selectivity of REMPI at $266 \mathrm{~nm}$ does allow the ability to detect these aromatic polymers without background interference from aliphatic polymers or additives in these polymer blends.

\section{ACKNOWLEDGMENTS}

We thank John Warren of Shell Development Co., Westhollow, TX for helpful suggestions during the course of this work. We also thank John Wronka of Bruker Instruments, Billerica, MA, for useful discussions regarding laser desorption and FAB-MS of polymers, David Jordan of Polysciences Inc. for the polystyrene data and Paul G. Rasmussen and Kevin Bouck of the University of Michigan for the polyamide samples. Special thanks to Ernest Thurber of the University of Michigan for invaluable discussions regarding polymer preparation and characterization. We gratefully acknowledge support by the Shell Development Co. and also received partial financial support by the National Science Foundation under Grant \# NSF CHE 8720401.

\section{REFERENCES}

1 D.M. Lubman, in D.M. Lubman (Ed.), Lasers and Mass Spectrometry, Oxford University Press, New York, 1990.

2 D.M. Lubman, Mass Spectrom. Rev., 7 (1989) 535, 559.

3 J.H. Hahn, R. Zenobi, J.L. Bada and R.N. Zare, Science, 239 (1988) 1523.

4 J. Grotemeyer and E.W. Schlag, Acc. Chem. Res., 22 (1989) 399.

5 R.B. Bernstein and L. Zandee, J. Chem. Phys., 70 (1979) 2574.

6 D.M. Lubman, R. Naaman and R.N. Zare, J. Chem. Phys., 71 (1979) 4192.

7 U. Boesl, H.J. Neusser and E.W. Schlag, J. Chem. Phys., 72 (1980) 4327.

8 L. Li and D.M. Lubman, Anal. Chem., 60 (1988) 2591.

9 J.R. Cable, M.J. Tubergen and D.H. Levy, J. Am. Chem. Soc., 110 (1988) 7349.

10 G. Meijer, M.S. de Vries, H.E. Hunziker and H.R. Wendt, Appl. Phys. B., in press.

11 T. Imasaka, K. Tashiro and N. Ishibashi, Anal. Chem., 61 (1989) 1530.

12 J. Grotemeyer, personal communication, ASMS, June 1990.

13 D. Feldmann, J. Kutzner, J. Laukemper, S. MacRobert and K.H. Welge, Appl. Phys. B., 44 (1987) 81.

14 R. Srinivasan and V. Mayne-Banton, Appl. Phys. Lett., 41 (1982) 576.

15 M. Stuke and R. Larciprete, Appl. Phys. B, 42 (1987) 181.

16 J.A. Syage and J.E. Wessel, Appl. Spectrosc. Rev., 24 (1988) 1. 
17 J.B. Pallix, V. Schuhle, C.H. Becker and D.L. Huestis, Anal. Chem., 61 (1989) 805.

18 D. Hrubowchak and N. Winograd, personal communication, 1990.

19 I.V. Blestos, D.M. Hercules, D. Van Leyden, E. Niehuis and A. Benninghoven, Springer Proc. Phys., 9 (1986) 74.

20 J.A. Warren, personal communication, Shell Development Co., 1990.

21 J.N. Kyranos, W.X. Zukas and P. Vouros, Macromolecules, 22 (1989) 1189. 